\title{
BMJ Open Leishmaniasis in Cameroon: what is known and is done so far? A protocol for systematic review
}

\author{
Linda Djune Yemeli, ${ }^{1,2}$ André Domche, ${ }^{3,4}$ Hugues C Nana Djeunga (D) , ,,4 \\ Cédric G Lenou Nanga, ${ }^{5}$ Earnest Njih Tabah, ${ }^{6}$ Georges B Nko'Ayissi, ${ }^{7}$ \\ Joseph Kamgno ${ }^{5,8}$
}

To cite: Djune Yemeli L, Domche A, Nana Djeunga HC, et al. Leishmaniasis in Cameroon: what is known and is done so far? A protocol for systematic review. BMJ Open 2021;11:e047530. doi:10.1136/ bmjopen-2020-047530

- Prepublication history and additional material for this paper are available online. To view these files, please visit the journal online (http://dx.doi. org/10.1136/bmjopen-2020047530).

Received 01 December 2020 Revised 16 March 2021 Accepted 23 March 2021

Check for updates

(C) Author(s) (or their employer(s)) 2021. Re-use permitted under CC BY-NC. No commercial re-use. See rights and permissions. Published by BMJ.

For numbered affiliations see end of article.

Correspondence to Dr Hugues C Nana Djeunga; nanadjeunga@crfilmt.org

\section{ABSTRACT}

Introduction The first visceral and cutaneous leishmaniasis cases were reported in Cameroon since more than six decades. However, interest in the disease has decreased over time and data on its epidemiology across the country are scanty. This systematic review aims to update data on what is known and done so far on leishmaniasis in Cameroon. Methods and analysis PubMed/MEDLINE, EMBASE and Web of Science will be searched from inception onwards. Grey literature will be identified through Google Scholar searches, dissertation databases and other relevant documents such as report of the National Control Program. Searches will be conducted between January and February 2021. All studies reporting endemicity, distribution, infecting species, vectors and reservoirs will be eligible. The main outcomes will be epidemiological data (infection rate, distribution, infecting species, vectors and animal reservoir), while the secondary outcomes will be the cases management (diagnostic, treatment reporting, intervention...). Two reviewers will independently screen eligible papers, and potential conflicts will be resolved by involving a third reviewer as an adjudicator. Methodological quality including bias will be appraised using a methodological quality critical appraisal checklist proposed in the Joanna Briggs Institute systematic review methods manual. A narrative synthesis will describe quality and content of the epidemiological evidence. Data on prevalence and vectors will be used to draw thematic maps of the distribution of leishmaniasis in Cameroon.

Ethics and dissemination This study will not require ethical approval as it will be based on already published or unpublished data. The final report of this review will be published in a peer-reviewed journal, and the outcomes will be used (1) as baseline information to design further studies that will help to better refine the epidemiological situation of leishmaniasis in Cameroon, and (2) to inform both programme managers and policy-makers of the situation of leishmaniasis in the country.

Systematic review registration This protocol was registered with the International Prospective Register of Systematic reviews (PROSPERO; registration number: CRD42020211864) database.

\section{INTRODUCTION}

Leishmaniasis is a complex vector-borne zoonotic disease caused by more than 20 species of an obligate intracellular parasitic

\section{Strengths and limitations of this study}

- To our knowledge, this article will be the second review on leishmaniasis in Cameroon, the first being done since 2001 . The results obtained will provide an update of the leishmaniasis situation in Cameroon, which is important for the development of better management strategy in the fight against this group of diseases.

- A narrative synthesis will be used to describe quality and content of the epidemiological evidence; a thematic map of the distribution of leishmaniasis in Cameroon will be drawn.

- A limit of this review could be the few number of published studies given that the disease is underexplored in Cameroon.

- There are often reports of suspicious cases with signs and symptoms of these diseases, but no confirmatory testing is carried out. This therefore represents a potential limitation to this study.

protozoa of the genus Leishmania, and transmitted by sand fly vectors of the genera Phlebotomus and Lutzomyia. ${ }^{1}$ Humans are infected when they share the same environment with a sand fly vector and reservoir hosts. There are different types of leishmaniasis according to the infecting species and clinical presentations. According to the 2010 WHO expert committee report, the different forms of leishmaniasis encountered in the old world are (1) visceral leishmaniasis (VL, also known as kala-azar, caused by $L$. donovani and $L$. infantum), (2) cutaneous leishmaniasis (CL, most frequently caused by L. tropica, L. major and L. aethiopica), (3) mucosal leishmaniasis (can be caused by any species), (4) diffused cutaneous leishmaniasis (caused by L. aethiopica) and (5) post kala-azar dermal leishmaniasis (present in all areas with $L$. donovani). ${ }^{2}$ While CL is the most common form of the disease, VL is the most serious and is almost always fatal if untreated. ${ }^{3}$ 
Leishmaniasis is highly heterogeneous in its distribution. In fact, while the incidence across a region may appear low, focal areas are intensely affected, leading to a high complexity in assessing the real incidence of the disease. ${ }^{45}$ Globally, the disease is endemic in all the six WHO regions, with 87 and 75 countries having reported at least one case of CL and VL, respectively. ${ }^{6}$ In 2015, an estimated 200000 new CL and 25000 new VL cases were reported worldwide. ${ }^{6}$

Both CL and VL have been described in Cameroon, and studies have revealed the presence of about 20 sand fly species. ${ }^{78}$ In fact, the first cases of CL and VL were described in 1930 and 1976 in the northern part of the country and in a hospital in Yaoundé, respectively. ${ }^{9} 10$ However, over time, studies on leishmaniasis are becoming scarce, and data are very scanty and poorly documented. Cameroon is currently classified among the countries with no data available on leishmaniasis. ${ }^{11}$ Indeed, Cameroon is classified by WHO as endemic to CL but with no available data on the number of cases, and appears among countries with previously reported VL cases with no available data on the number of cases. ${ }^{6}$ Since the systematic review on the situation of leishmaniasis in Cameroon carried out in $2001,{ }^{9}$ and the leishmaniasis country profile established by WHO in 2012, ${ }^{12}$ no update has been made to allow the establishment of new management strategies against this group of diseases. There is therefore an urgent need to perform a situation analysis of leishmaniasis in Cameroon.

\section{Objective}

This systematic review aims to document the infection rate, circulating species, vectors and reservoirs of leishmaniasis as well as data on control/management strategies in Cameroon. This will help in updating the situation of leishmaniasis in Cameroon, and ultimately helps defining/refining control strategies and reinforcing advocacy.

\section{Review questions}

By documenting data on what is known and done so far on leishmaniasis in Cameroon, this review will help in answering the following questions:

1. What are the circulating species and the clinical presentations?

2. What are the vectors responsible of the transmission of leishmaniasis?

3. What are the non-human reservoirs of leishmaniasis?

4. How is the diagnostic of leishmaniasis conducted?

5. What is the level of endemicity and distribution of leishmaniasis?

6. How is the control of leishmaniasis organised?

\section{METHODS AND ANALYSIS}

This systematic review protocol was written in accordance with reporting guidance provided by the Preferred Reporting Items for Systematic Reviews and Meta-Analyses Protocols (PRISMA-P) statement ${ }^{13}$ (see
PRISMA-P checklist as online supplemental file 1). The registration process of the protocol with the Prospective Register of Systematic reviews (PROSPERO) database is done (registration process ongoing in PROSPERO). The review process will be conducted between January and February 2021.

\section{Eligibility criteria}

There will be no restrictions regarding the date of the study. Any type of study, either published or not, on Leishmania species, endemicity, distribution, clinical presentation, co-infection (HIV or any other infection), vectors and reservoirs in Cameroon will be eligible for this review. Since leishmaniasis belongs to case management Neglected Tropical Diseases group, case reports and health facilities' data will also be considered in this review. In addition, study or reports on control/management as well as surveillance will be of interest. However, systematic reviews or meta-analyses will be excluded. The search languages will be English and French, the Cameroon official languages in which almost all publications are done.

\section{Information sources and search strategies}

PubMed/MEDLINE, EMBASE and Web of Science will be searched, from their inception onwards, to identify relevant articles. Grey literature will be identified through search in Google Scholar and other relevant documents such as dissertation databases and government/control programme reports. The combination of keywords to use in the search strategy will be "leishmaniose" OR "leishmaniasis" OR "Kala-azar" OR "black fever" OR "fièvre noire" OR "Leishmania" OR "sandflies" OR "mouche du sable" OR "phlebotomine" OR "Phlebotomus" AND "Cameroun" OR "Cameroon" (online supplemental file 2). Authors of primary publications or aggregated data and stakeholders involved in research and/or control of leishmaniasis in Cameroon (National Buruli Ulcer, Leprosy, Yaws and Leishmaniasis Control Program) will be contacted to request for unpublished data and/or resources (reports, datasets) relevant for this study.

\section{Study selection}

Two independent reviewers will first screen titles and abstracts against eligibility criteria to identify studies that can be potentially included in this review. Studies whose titles and abstracts will give indication that they contain any relevant information on the topic will be included. Full texts of articles deemed potentially relevant will then be retrieved and assessed by the two independent reviewers for the compliance with eligibility criteria. Finally, data will be extracted using a purpose-built Microsoft Office Excel spreadsheet. In case of disagreements between the two independent reviewers, a third one will be involved as an adjudicator, either by consensus or by discussion.

\section{Data extraction and management}

A reference manager software (EndNote/Zotero) will be used to manage the retrieval of literature and to screen for 
and exclude duplicates. This will be done first automatically using the "find duplicate" or "de-duplication" function under EndNote or Zotero, respectively, by comparing the title or various combinations of the author(s), year, secondary title, volume, issue and page numbers. In the second instance, the records of suspected duplicates will be visually inspected.

Full texts will be read, and Excel spreadsheet will be used for data extraction. The following items will be extracted: (a) title, (b) years of publication, (c) author names, (d) location of the study (regions, health areas, communities), (e) type of environment (forest, savannah, forest-savannah mosaic, type of vegetation), (f) place of report (communities, school or hospital for case report), (g) date of data collection, (h) type of study (is the study on human, vectors or animal reservoirs?), (i) study design, (j) diagnostic methods, (k) Leishmania species, (l) vector species, (m) potential reservoirs, (n) treatment provided and (o) geographical coordinates (latitude, longitude and altitude).

\section{Outcomes}

The main outcomes will be (1) the prevalence of leishmaniasis, (2) the distribution throughout the country, (3) the infecting species and their distribution, (4) the vectors responsible of transmission and (5) the animal reservoir species. In addition, the secondary outcomes will be the management of cases (diagnostic, treatment, reporting, intervention...). We therefore expected at the end of this study to demonstrate that although data on leishmaniasis are scanty in Cameroon, the disease is a public health concern. This will further serve (1) as a basis to design studies that will help to better refine the epidemiological situation of leishmaniasis in Cameroon, and (2) to inform both programme managers and policymakers of the situation of leishmaniasis in the country.

\section{Data analysis}

Data will be recorded as prevalence with 95\% CIs. ${ }^{14}$ Prevalence of infection estimates will be stratified according to gender, age, geographical location and year of publication. Chi-square test will be used to compare the prevalence of leishmaniasis between different data collection time points/periods. Data on prevalence and vectors will be used to draw a thematic map of the distribution of leishmaniasis in Cameroon using a geographical information system (GIS) software (ArcGIS, V.10.2; ESRI)

\section{Assessment of risk of bias}

The risk of bias of primary observational studies will be evaluated using a methodological quality critical appraisal checklist proposed in the Joanna Briggs Institute systematic review methods manual. ${ }^{15}$ We will also not include studies with aggregated data by community/village.

\section{Confidence in cumulative evidence}

The quality of the evidence will be judged using the Grading of Recommendations Assessment, Development and Evaluation (GRADE) approach. ${ }^{16}$ Evidence quality assessment will be performed for each outcome. The grades of evidence will be defined into four categories and adjudicated as "high" (further research is unlikely to change our confidence in the estimate of effect), "moderate" (further research is likely to have an important impact on our confidence in the estimate of effect and may change the estimate), "low" (further research is very likely to have an important impact on our confidence in the estimate of effect and is likely to change the estimate) and "very low" (any estimate of effect is very uncertain). ${ }^{16}$ The confidence in evidence will be discussed among authors, and a narrative synthesis of the results will be provided as some degree of heterogeneity is expected.

\section{Presentation and reporting of results}

A flow chart will be used to demonstrate the study selection process. Table or plots will be used to represent qualitative/quantitative variables when appropriate. Data on prevalence, infective species and vectors will be used to draw a thematic map of the distribution of leishmaniasis in Cameroon.

\section{Patient and public involvement}

No patient involved.

\section{Amendments to protocol}

After approval of the protocol, any important amendments will be documented in the final publication. The date, rationale and description of each change will be provided. If necessary, these amendments will be registered with PROSPERO.

\section{ETHICS AND DISSEMINATION}

This proposed study will not require ethical approval as it will be based on already existing published or unpublished data. The final report of this review will be published in a peer-reviewed journal, and the outcomes will be used (1) as baseline information to design further studies that will help to better refine the epidemiological situation of leishmaniasis in Cameroon, and (2) to inform both programme managers and policy-makers of the situation of leishmaniasis in the country.

\footnotetext{
Author affiliations

${ }^{1}$ Molecular Parasitology and Genetic Epidemiology, Centre for Research on Filariasis and other Tropical Diseases (CRFilMT), Yaounde, Cameroon

${ }^{2}$ Molecular Diagnosis Research Group, Biotechnology Centre, University of Yaounde I, Yaounde, Cameroon

${ }^{3}$ Entomology and Vector-Borne Diseases, Centre for Research on Filariasis and other Tropical Diseases (CRFilMT), Yaounde, Cameroon

${ }^{4}$ Department of Animal Biology and Physiology, Faculty of Science, University of Yaounde I, Yaounde, Cameroon

${ }^{5}$ Epidemiology and Biostatistics, Centre for Research on Filariasis and other Tropical Diseases (CRFilMT), Yaounde, Cameroon

${ }^{6}$ National Buruli Ulcer, Leprosy, Yaws and Leishmaniasis Control Program, Ministry of Public Health, Yaounde, Cameroon

${ }^{7}$ National Neglected Tropical Diseases Coordination Unit, Ministry of Public Health, Yaounde, Cameroon
} 
${ }^{8}$ Department of Public Health, Faculty of Medicine and Biomedical Sciences, University of Yaounde I, Yaounde, Cameroon

Twitter Hugues C Nana Djeunga @Nana_Djeunga

Contributors LDY, AD, HCND, CGLN, ENT, GBN and JK contributed intellectually to the development of the present protocol. LDY, AD and HCND prepared the first draft of the manuscript. LDY, AD, HCND, CGLN, ENT, GBN and JK reviewed and approved the final version of the manuscript. HCND and JK are the study guarantors.

Funding The authors have not declared a specific grant for this research from any funding agency in the public, commercial or not-for-profit sectors.

Competing interests None declared.

Patient consent for publication Not required.

Provenance and peer review Not commissioned; externally peer reviewed.

Supplemental material This content has been supplied by the author(s). It has not been vetted by BMJ Publishing Group Limited (BMJ) and may not have been peer-reviewed. Any opinions or recommendations discussed are solely those of the author(s) and are not endorsed by BMJ. BMJ disclaims all liability and responsibility arising from any reliance placed on the content. Where the content includes any translated material, BMJ does not warrant the accuracy and reliability of the translations (including but not limited to local regulations, clinical guidelines, terminology, drug names and drug dosages), and is not responsible for any error and/or omissions arising from translation and adaptation or otherwise.

Open access This is an open access article distributed in accordance with the Creative Commons Attribution Non Commercial (CC BY-NC 4.0) license, which permits others to distribute, remix, adapt, build upon this work non-commercially, and license their derivative works on different terms, provided the original work is properly cited, appropriate credit is given, any changes made indicated, and the use is non-commercial. See: http://creativecommons.org/licenses/by-nc/4.0/.

ORCID iD

Hugues C Nana Djeunga http://orcid.org/0000-0002-4496-6048

\section{REFERENCES}

1 World Health Organization. Control of the leishmaniases: report of a WHO Expert Committee. Weekly Epidemiological Record= Relevé épidémiologique hebdomadaire 1991;66:88.
2 World Health Organization. Report of a meeting of the WHO Expert Committee on the Control of Leishmaniases, 22-26 March 2010. WHO Technical Report Series. Geneva, Switzerland: World Health Organization, 2010.

3 Colmenares M, Kar S, Goldsmith-Pestana K, et al. Mechanisms of pathogenesis: differences amongst Leishmania species. Trans $R$ Soc Trop Med Hyg 2002;96 Suppl 1:S3-7.

4 Bern C, Hightower AW, Chowdhury R, et al. Risk factors for kala-azar in Bangladesh. Emerg Infect Dis 2005;11:655-62.

5 Bern C, Maguire JH, Alvar J. Complexities of assessing the disease burden attributable to leishmaniasis. PLoS Negl Trop Dis 2008;2:e313.

6 mondiale de la Santé O, World Health Organization. Global leishmaniasis update, 2006-2015: a turning point in leishmaniasis surveillance-Le point sur La situation mondiale de la leishmaniose, 2006-2015: un tournant dans La surveillance de la maladie. Weekly Epidemiological Record= Relevé épidémiologique hebdomadaire 2017;92:557-65.

7 Dondji B, Duhlinska DD, Same-Ekobo A. Species composition of the phlebotomine sandfly fauna (Diptera: Phlebotominae) in Mokolo region, Northern Cameroon. Insect Science and Its Application 2000;20:221-6.

8 Tateng AN, Payne VK, Ngouateu OB, et al. Inventory and taxonomy of phlebotomine sand flies of the Mokolo leishmaniasis focus, Northern Cameroon, with description of new Sergentomyia taxa (Diptera: Psychodidae). Acta Trop 2019;194:172-80.

9 Dondji B. [Leishmaniasis and phlebotomus of Cameroon: review of current data]. Bull Soc Pathol Exot 2001;94:277-9.

10 Deniau M, Mbede J, Obama M. Premier Cas confirmé de leishmaniose viscérale Au Cameroun. Bull Soc Fr Parasitol 1986;4:197-200.

11 Gyapong J, Boatin B. Neglected tropical diseases-sub-Saharan Africa. Springer, 2016.

12 Alvar J, Vélez ID, Bern C, et al. Leishmaniasis worldwide and global estimates of its incidence. PLoS One 2012;7:e35671.

13 Moher D, Shamseer L, Clarke M, et al. Preferred reporting items for systematic review and meta-analysis protocols (PRISMA-P) 2015 statement. Syst Rev 2015;4:1.

14 Brown LD, Cai TT, DasGupta A. Interval estimation for a binomial proportion. Statistical science 2001:101-17.

15 Munn Z, Moola S, Lisy K. Chapter 5: systematic reviews of prevalence and incidence. In: Joanna Briggs Institute Reviewer's Manual. 37. The Joanna Briggs Institute, 2017.

16 Atkins D, Best D, Briss PA, et al. Grading quality of evidence and strength of recommendations. BMJ 2004;328:1490. 